\title{
Application of High Speed Cameras for 4D Data Collection in S/TEM
}

\author{
Anahita Pakzad ${ }^{1}$, Cory Czarnik ${ }^{1}$, Roy Geiss ${ }^{2}$, Everett Jackson ${ }^{2}$, David Mastronarde ${ }^{3}$ \\ 1. Gatan Inc., Pleasanton, CA, USA. \\ 2. Colorado State University, Chemistry Department, Fort Collins, CO, USA. \\ 3. University of Colorado, MCDB, Boulder, CO, USA.
}

Digital imaging in transmission electron microscope (TEM) has come a long way since 1982, when the first experimental camera based on a charge coupled device (CCD) was reported [1]. Some of the major developments in the past fifteen years include use of complementary metal-oxide semiconductor (CMOS) devices, and subsequently improvements in camera sensitivity, detective quantum efficiency (DQE) and speed. Some of the latest applications of high speed CMOS cameras from in situ experiments, electron tomography (ET) and four dimensional scanning TEM (4D-STEM) diffraction experiments will be presented here.

Historically, image recording in in situ TEM started with manual static image acquisition on film, while later images of a TV camera were recorded onto a videotape [2], subsequently followed by use of CCD cameras to record "real-time" digital images. Until just a few years ago the time resolution of CCD recorded in situ datasets was limited to $1 / 30 \mathrm{~s}$, and the recording was a "picture" of the data in each pixel, not the actual values (data) recorded in each pixel. Such time resolution is not sufficient for close observation of rapid dynamics in most TEM experiments, and this limitation was one of the driving forces in the development of high speed CMOS cameras available today, currently offering performance of many 100's of fps resolution. In this work, we will show experimental results in different materials systems that are uniquely enabled by these high speed cameras.

ET is used for three dimensional (3D) studies of nanoscale materials in a TEM, and it consists of acquisition of a series of images of the specimen in different viewing directions [3]. The resolution of the 3D reconstruction is directly affected by the tilt range and tilt increment in an ET experiment. On the other hand, in cases where specimens are electron-beam-sensitive or change as a function of time, there is a restriction on the number of projections that can be recorded. A high speed camera is therefore very beneficial in low dose ET and 3D time-resolved studies of dynamic processes in a TEM. We will present high speed ET datasets collected, using high speed and high DQE cameras, while the microscope goniometer was continuously tilting. This technique reduces the total data collection time from several tens of minutes to a few minutes, and improves the resolution of the $3 \mathrm{D}$ reconstructed volume for thicker samples by reducing the tilt increment from 1-3 degrees to a small fraction of a degree.

STEM diffraction imaging is an accurate analysis technique to acquire structure, strain, and texturerelated information. In this method either a parallel or a convergent electron beam having a probe size as small as $0.2 \mathrm{~nm}$ is used to generate diffraction patterns. These patterns can be used to characterize individual nano particles, defects and interfaces and allow accurate measurements of strain and crystal orientation. 4D-STEM diffraction data is collected by scanning the electron probe on the specimen and collecting a diffraction pattern at each pixel of the scan. One of the main challenges with this technique has been the limitation of the data collection speed. Conventional CCD cameras are limited to about 30 frames per second (fps), which limits the number of diffraction patterns that can be collected in a reasonable amount of time. This is even more challenging in cases where specimens are beam sensitive 
and/or drift. In such cases by the time data collection is complete, the sample has experienced radiation damaged or moved out of the field of view. We will compare 4D-STEM datasets collected with both high speed CMOS and conventional CCD cameras, to show how newly developed CMOS systems with superior DQE and speed can benefit such experiments.

In Figure 1a below, we show segments from two images captured at the onset of a phase transformation in a $\mathrm{ZnSb}$ thin film with $0.05 \mathrm{sec}$ between images. A live FFT is valuable for evaluating the high speed amorphous / crystalline transformation. In Figure 1b, we show CBED patterns from outside and inside a vacancy dislocation loop in a copper foil. In Figure 1c, we show two images from a high speed, one degree per second, tilting experiment using arrays of gold nanoparticles. The two images are approximately 60 degrees apart. The full 120 degree tilting experiment was done at $25 \mathrm{kX}$ in the TEM in 110 seconds. The reduction of such a data stream as a tomogram will be presented.

\section{References:}

[1] PTE Roberts, JN Chapman and AM MacLeod, Ultramicroscopy 8 (1982), p. 385.

[2] J Vetrano, SM Bruemmer and IM Robertson, MRS Symposium Proc. 404 (1996), p. 177

[3] PA Midgley et al, Chem Soc Rev 36 (2007), p. 1477
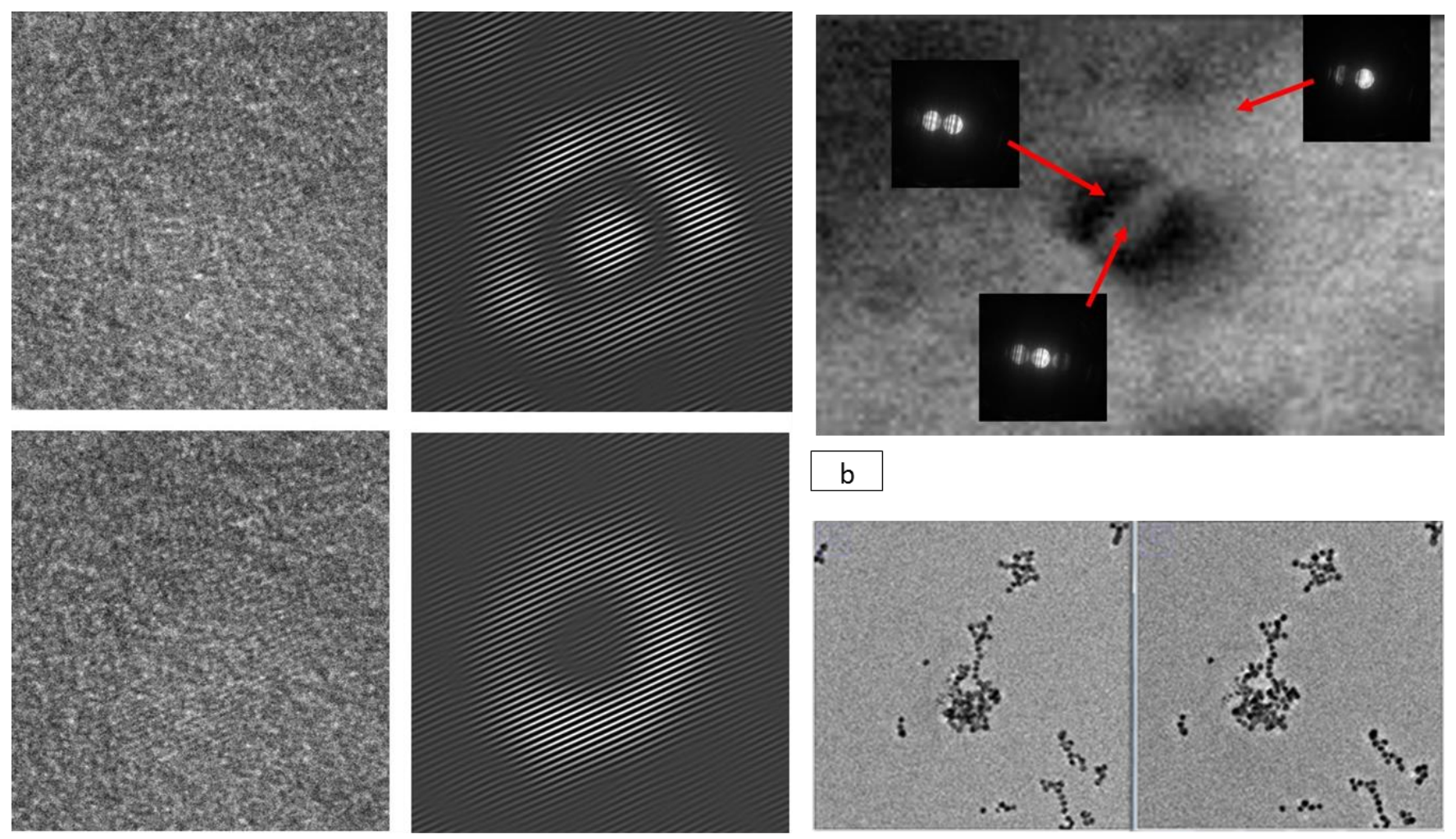

a

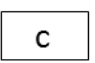

Figure 1. (a) Images and IFFTs taken $0.05 \mathrm{sec}$ apart during heating of $\mathrm{ZnSb}$ thin film. It is very hard to see the structure in the direct image, but with the use of a live FFT one can detect rapid changes. The IFFTs show clearly the changes in crystallinity taking place. Each image is $15 \mathrm{~nm}$ wide. (b) CBED patterns from a prismatic dislocation loop in a copper foil. Image width is $100 \mathrm{~nm}$. (c) Pair of images 60 degrees apart in high speed tilt experiment. Each image is $575 \mathrm{~nm}$ wide. 